\title{
Pseudo-State Sliding Mode Control of Fractional SISO Nonlinear Systems
}

\author{
Bao Shi, Jian Yuan, and Chao Dong \\ Institute of Systems Science and Mathematics, Naval Aeronautical and Astronautical University, Yantai, Shandong 264001, China \\ Correspondence should be addressed to Jian Yuan; yuanjianscar@gmail.com
}

Received 20 August 2013; Revised 6 October 2013; Accepted 6 October 2013

Academic Editor: J. A. Tenreiro Machado

Copyright (C) 2013 Bao Shi et al. This is an open access article distributed under the Creative Commons Attribution License, which permits unrestricted use, distribution, and reproduction in any medium, provided the original work is properly cited.

\begin{abstract}
This paper deals with the problem of pseudo-state sliding mode control of fractional SISO nonlinear systems with model inaccuracies. Firstly, a stable fractional sliding mode surface is constructed based on the Routh-Hurwitz conditions for fractional differential equations. Secondly, a sliding mode control law is designed using the theory of Mittag-Leffler stability. Further, we utilize the control methodology to synchronize two fractional chaotic systems, which serves as an example of verifying the viability and effectiveness of the proposed technique.
\end{abstract}

\section{Introduction}

Fractional calculus has a long history of three hundred years, over which a firm theoretical foundation has been established. In the past few decades, with deep understanding of the power of fractional calculus and rapid development of computer technology, an enormous number of interesting and novel applications have emerged in physics, chemistry, engineering, finance, and other sciences [1-3]. In particular, engineers and scientists from various fields have developed plenty of fractional dynamical systems, that is, systems which are better characterized by noninteger order mathematics models. As has been stated in [3], a suitable way to more efficient control of fractional dynamical systems is to design fractional controllers.

Several pioneering attempts to develop fractional control methodologies have been made, such as TID controller [4], CRONE controller [5], fractional PID controller [6], and fractional lead-lag compensator [7]. Basic ideas and technical formulations of the above four fractional control schemes with comparative comments have been presented in [8].

Very recently, by applying fractional calculus to advanced nonlinear control theory, several fractional nonlinear control schemes have been proposed, such as fractional sliding mode control, fractional adaptive control, and fractional optimal control. Exactly, to design fractional sliding mode controls, various fractional sliding surfaces have been constructed in
[9-21]. In particular, adaptive sliding mode controls have been proposed in [12, 19-21], fractional terminal sliding mode controls in $[15,16]$, and sliding mode controls for linear fractional systems with input and state delays in [17]. In [22], the authors have presented two ideas to extend the conventional model reference adaptive control (MRAC) by using fractional parameter adjustment rule and fractional reference model. In [23], a fractional model reference adaptive control algorithm for SISO plants has been proposed, which can guarantee the stability and ability to reject disturbances. In [24-26], adaptive controllers have been designed to control and synchronize fractional chaotic systems. In [27], Agrawal has proposed a general formulation for a class of fractional optimal control problems, which is specialized for a system with quadratic performance index subject to a fractional system dynamic constraint. In [28], the authors have generalized the optimality conditions of [27]. Besides the above fractional control methodologies, fractional optimal synergetic control has been presented in [29] and active disturbance rejection control in [30].

Motivated by the above contributions, this paper proposes a sliding mode control design for fractional SISO nonlinear systems in the presence of model inaccuracies. By constructing a stable fractional sliding mode surface on the basis of Routh-Hurwitz conditions, a sliding mode control law is designed. Further, stability analysis is performed using Mittag-Leffler stability theory. Comparing this with methods 
in the previous papers, we utilize the fractional derivative of the sliding mode surface instead of first-order derivative, to obtain the equivalent control law. Moreover, to carry out the stability analysis of the closed-loop fractional nonlinear system, we use the fractional derivative of the Lyapunov function candidate in terms of Theorem 2 in [18].

The rest of the paper is organized as follows. Section 2 reviews some basic definitions for fractional calculus. Section 3 proposes the sliding control design for fractional SISO nonlinear systems. Numerical simulations of synchronization of the fractional Genesio-Tesi system and the fractional Arneodo system are presented in Section 4. Finally, Section 5 concludes this paper with some remarks on future study.

\section{Basic Definitions for Fractional Calculus}

Fractional calculus is a generalization of integration and differentiation to noninteger order fundamental operator ${ }_{a} D_{t}^{\alpha}$, where $a$ and $t$ are the bounds of the operation and $a \in R$. The continuous integrodifferential operator is defined as [1]

$$
{ }_{a} D_{t}^{\alpha}= \begin{cases}\frac{d^{\alpha}}{d t^{\alpha}} & \alpha>0 \\ 1 & \alpha=0 \\ \int_{a}^{t}(d \tau)^{\alpha} & \alpha<0 .\end{cases}
$$

The three most frequently used definitions for fractional calculus are the Grünwald-Letnikov definition, the RiemannLiouville definition, and the Caputo definition [1-3].

Definition 1. The Grünwald-Letnikov derivative definition of order $\alpha$ is described as

$$
{ }_{a} D_{t}^{\alpha} f(t)=\lim _{h \rightarrow 0} \frac{1}{h^{\alpha}} \sum_{j=0}^{\infty}(-1)^{j}\left(\begin{array}{c}
\alpha \\
j
\end{array}\right) f(t-j h) .
$$

Definition 2. The Riemann-Liouville derivative definition of order $\alpha$ is described as

$$
{ }_{a} D_{t}^{\alpha} f(t)=\frac{1}{\Gamma(n-\alpha)} \frac{d^{n}}{d t^{n}} \int_{a}^{t} \frac{f(\tau) d \tau}{(t-\tau)^{\alpha-n+1}}, \quad n-1<\alpha<n .
$$

However, applied problems require definitions of fractional derivatives allowing the utilization of physically interpretable initial conditions, which contains $f(a), f^{\prime}(a)$, and so forth. Unfortunately, the Riemann-Liouville approach fails to meet this practical need. It is M. Caputo who solved this conflict.

Definition 3. The Caputo definition of fractional derivative can be written as

$$
{ }_{a} D_{t}^{\alpha} f(t)=\frac{1}{\Gamma(n-\alpha)} \int_{a}^{t} \frac{f^{(n)}(\tau) d \tau}{(t-\tau)^{\alpha-n+1}}, \quad n-1<\alpha<n .
$$

In the rest of the paper, we use the Caputo approach to describe the fractional systems and the Grünwald-Letnikov approach to propose numerical simulations. To simplify the notation, we denote the fractional-order derivative of order $\alpha$ as $D^{\alpha}$ instead of ${ }_{0} D_{t}^{\alpha}$ in this paper.

\section{Sliding Control of Fractional SISO Nonlinear Systems}

Consider fractional SISO nonlinear systems

$$
\begin{gathered}
D^{\alpha} x_{i}=x_{i+1}, \quad i=1,2, \ldots, n-1, \\
D^{\alpha} x_{n}=f(\mathbf{x})+g(\mathbf{x}) u, \\
y=x_{1},
\end{gathered}
$$

where $\alpha \in(0,1]$ is the order of the dynamic system, $\mathbf{x}=$ $\left(x_{1}, x_{2} \ldots, x_{n}\right)^{T} \in \mathbb{R}^{n}$ is denoted as state vector, and the scalar $u, y \in R$ are system's input and output, respectively. The dynamic $f(\mathbf{x})$ (possibly nonlinear or time varying) is not exactly known, but estimated as $\widehat{f}(\mathbf{x})$. The control gain $g(\mathbf{x})$ (possibly time varying or state dependent) is an unknown function.

Assumption 4. The estimation error on $f(\mathbf{x})$ is assumed to be bounded by some known function $F(\mathbf{x})$ :

$$
|f(\mathbf{x})-\widehat{f}(\mathbf{x})| \leq F(\mathbf{x}) .
$$

Assumption 5. $g(\mathbf{x})$ is assumed to be bounded by

$$
0<g_{\min }(\mathbf{x}) \leq g(\mathbf{x}) \leq g_{\max }(\mathbf{x}),
$$

where both $g_{\min }(\mathbf{x})$ and $g_{\max }(\mathbf{x})$ are known functions.

Assumption 6. The desired tracking signal is denoted as $y_{d}(t)$, and it is assumed that $y_{d}(t) \in C^{n}[0, \infty)$ and $y_{d}(t)$, $D^{\alpha} y_{d}(t), \ldots, D^{n \alpha} y_{d}(t)$ are all bounded.

In this paper, our goal is to design a suitable fractional sliding mode controller to make the output $y$ of the system (5) track the desired signal $y_{d}$; that is,

$$
\lim _{t \rightarrow \infty}\left(y-y_{d}\right)=0 \text {. }
$$

Now we are ready to give the design steps.

Firstly, the tracking error is defined as

$$
\tilde{x}=y-y_{d} .
$$

Then, the tracking error vector is defined as

$$
\begin{aligned}
\widetilde{\mathbf{x}} & =x-\left(y_{d}, D^{\alpha} y_{d}, \ldots, D^{(n-1) \alpha} y_{d}\right)^{T} \\
& =\left(\tilde{x}, D^{\alpha} \tilde{x}, \ldots, D^{(n-1) \alpha} \tilde{x}\right)^{T} .
\end{aligned}
$$

A fractional sliding surface is proposed as

$$
s(t)=c_{1} \tilde{x}+c_{2} D^{\alpha} \tilde{x}+\cdots+c_{n-1} D^{(n-2) \alpha} \tilde{x}+D^{(n-1) \alpha} \tilde{x},
$$

where the polynomial $P(p)=c_{1}+c_{2} p+\cdots+c_{n-1} p^{n-2}+p^{n-1}$ satisfies Routh-Hurwitz conditions for fractional differential equations [31]. 
The fractional sliding surface (11) can be rewritten as

$$
\begin{aligned}
s(t) & =D^{(n-1) \alpha} \tilde{x}+\sum_{i=1}^{n-1} c_{i} D^{(i-1) \alpha} \tilde{x} \\
& =D^{(n-1) \alpha} y-D^{(n-1) \alpha} y_{d}+\sum_{i=1}^{n-1} c_{i} D^{(i-1) \alpha} \tilde{x} \\
& =D^{(n-1) \alpha} x_{1}-D^{(n-1) \alpha} y_{d}+\sum_{i=1}^{n-1} c_{i} D^{(i-1) \alpha} \tilde{x} \\
& =x_{n}-D^{(n-1) \alpha} y_{d}+\sum_{i=1}^{n-1} c_{i} D^{(i-1) \alpha} \tilde{x} .
\end{aligned}
$$

Taking its $\alpha$ th order derivative with respect to time yields

$$
D^{\alpha} s(t)=D^{\alpha} x_{n}-D^{n \alpha} y_{d}+\sum_{i=1}^{n-1} c_{i} D^{i \alpha} \tilde{x}
$$

Substituting the second equation of system (5) into (13), one has

$$
D^{\alpha} s(t)=f(x)+g(x) u-D^{n \alpha} y_{d}+\sum_{i=1}^{n-1} c_{i} D^{i \alpha} \tilde{x}
$$

In terms of Assumption 5, one of the estimated values of $g(\mathbf{x})$ can be chosen as

$$
\widehat{g}(\mathbf{x})=\sqrt{g_{\min }(\mathbf{x}) g_{\max }(\mathbf{x})} .
$$

Bounds of $g(x)$ are written as

$$
\beta^{-1} \leq \frac{\widehat{g}(\mathbf{x})}{g(\mathbf{x})} \leq \beta
$$

where $\beta=\sqrt{g_{\max } / g_{\min }}$.

Design the control law as

$$
\begin{aligned}
u(t)=\frac{1}{\widehat{g}(\mathbf{x})}\left[-\widehat{f}(\mathbf{x})+D^{n \alpha} y_{d}-\sum_{i=1}^{n-1} c_{i} D^{i \alpha} \tilde{x}\right. \\
\quad-k(\mathbf{x}) \cdot \operatorname{sign}(s)-\sigma s] \\
=\widehat{u}_{e q}(t)+u_{r}(t),
\end{aligned}
$$

where $\widehat{u}_{e q}(t)=(1 / \widehat{g}(\mathbf{x}))\left[-\widehat{f}(\mathbf{x})+D^{n \alpha} y_{d}-\sum_{i=1}^{n-1} c_{i} D^{i \alpha} \tilde{x}\right], u_{r}(t)=$ $(1 / \widehat{g}(\mathbf{x}))[-k(\mathbf{x}) \cdot \operatorname{sign}(s)-\sigma s], k(\mathbf{x}) \geq \beta(F(\mathbf{x})+\eta)+\beta(\beta-1)\left|\widehat{u}_{e q}\right|$, and $\eta$ is a positive constant.

To ensure the stability of the fractional system (5), we have the following theorem.

Theorem 7. Under Assumptions 4-6, the fractional SISO nonlinear system (5) can be controlled using the sliding mode control law (17) with the fractional sliding surface (11).
Proof. Consider the following candidate Lyapunov function:

$$
V(t)=[s(t)]^{2}
$$

where $s(t)$ is the fractional sliding surface (11) constructed previously.

Taking its $\alpha$ th order derivative with respect to time along with the fractional sliding surface (11), one has

$$
\begin{aligned}
D^{\alpha} V & =s \cdot D^{\alpha} s+\sum_{j=1}^{\infty} \frac{\Gamma(1+q)}{\Gamma(1+j) \Gamma(1-j+q)} s^{(j)} D^{q-j} s \\
& \leq s \cdot D^{\alpha} s+\rho,
\end{aligned}
$$

where $\rho$ is assumed to be an arbitrarily large positive constant which is a bound on the series of (19).

Substituting (14) into (19) yields

$$
D^{\alpha} V \leq s\left[f(\mathbf{x})+g(\mathbf{x}) u-D^{n \alpha} y_{d}+\sum_{i=1}^{n-1} c_{i} D^{i \alpha} \tilde{x}\right]+\rho .
$$

Substituting the control law (17) into (20) gives

$$
\begin{aligned}
D^{\alpha} V \leq s & {\left[f-g \widehat{g}^{-1} \widehat{f}+\left(1-g \widehat{g}^{-1}\right)\left(-D^{n \alpha} y_{d}+\sum_{i=1}^{n-1} c_{i} D^{i \alpha} \tilde{x}\right)\right.} \\
& \left.-g \widehat{g}^{-1} k \operatorname{sign}(s)\right]-\sigma s^{2}+\rho \\
= & s\left[(f-\widehat{f})+\widehat{f}-g \widehat{g}^{-1} \widehat{f}+\left(1-g \widehat{g}^{-1}\right)\right. \\
& \left.\times\left(-D^{n \alpha} y_{d}+\sum_{i=1}^{n-1} c_{i} D^{i \alpha} \tilde{x}\right)-g \widehat{g}^{-1} k \operatorname{sign}(s)\right] \\
& -\sigma s^{2}+\rho \\
= & s\left[(f-\widehat{f})+\left(g \widehat{g}^{-1}-1\right) \widehat{u}_{e q}-g \widehat{g}^{-1} k \operatorname{sign}(s)\right] \\
& -\sigma s^{2}+\rho .
\end{aligned}
$$

Substituting the estimation error (6), the equivalent control law $\widehat{u}_{e q}$, and control gain $k$ of (17) into (21), one derives

$$
D^{\alpha} V \leq-\eta|s|-\sigma s^{2}+\rho<-\sigma V+\rho,
$$

which implies that the sliding mode dynamic is globally stable and the tracking error vector (10) converges to zero according to Theorem 2 in [18]. This proves that the fractional SISO nonlinear system (5) can be controlled using the sliding mode control law (17) with the fractional sliding surface (11).

\section{Numerical Simulations}

In this section, we apply the fractional sliding mode control method proposed in Section 3 to deal with the problem of 


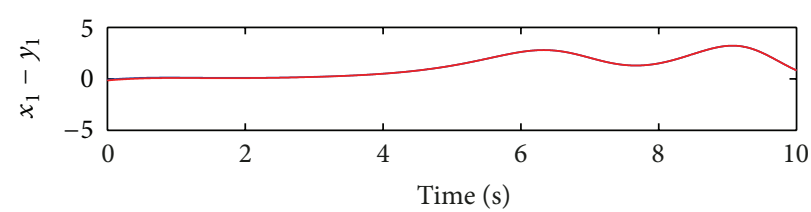

(a)

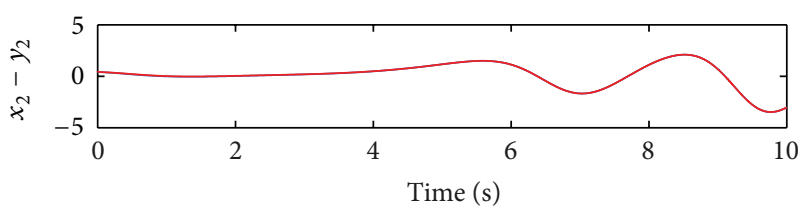

(b)

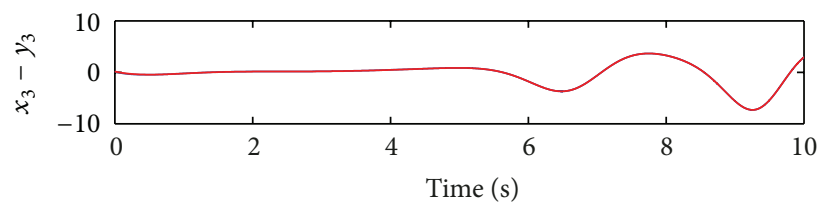

(c)

Figure 1: Synchronization of the fractional Arneodo system and the fractional Genesio-Tesi system with the control input (27) (red line represents the trajectories of the drive system, while blue line represents the trajectories of the response system).

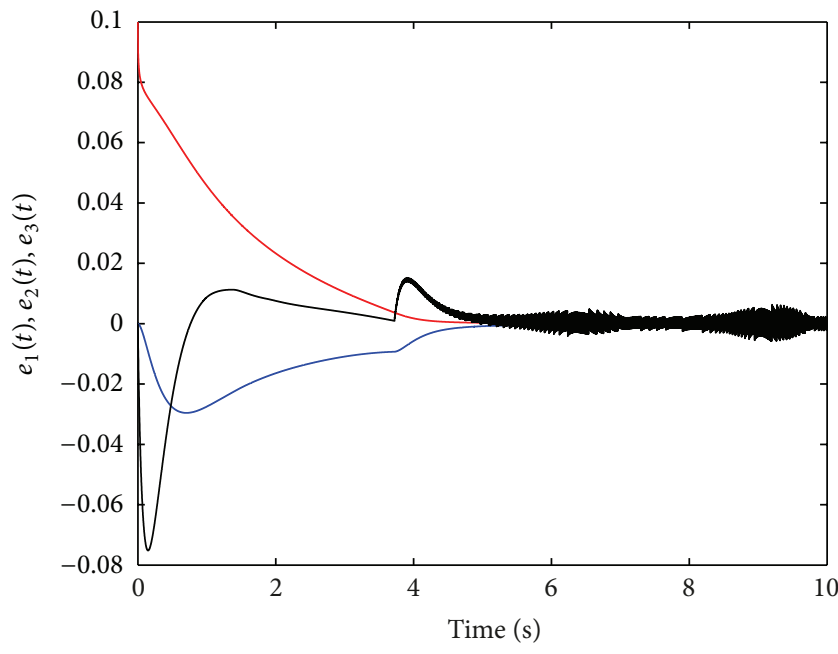

FIGURE 2: Synchronization errors with the control input (27) (red line represents the first error state $e_{1}$, blue line represents the second error state $e_{2}$, and black line represents the third error state $e_{3}$ ).

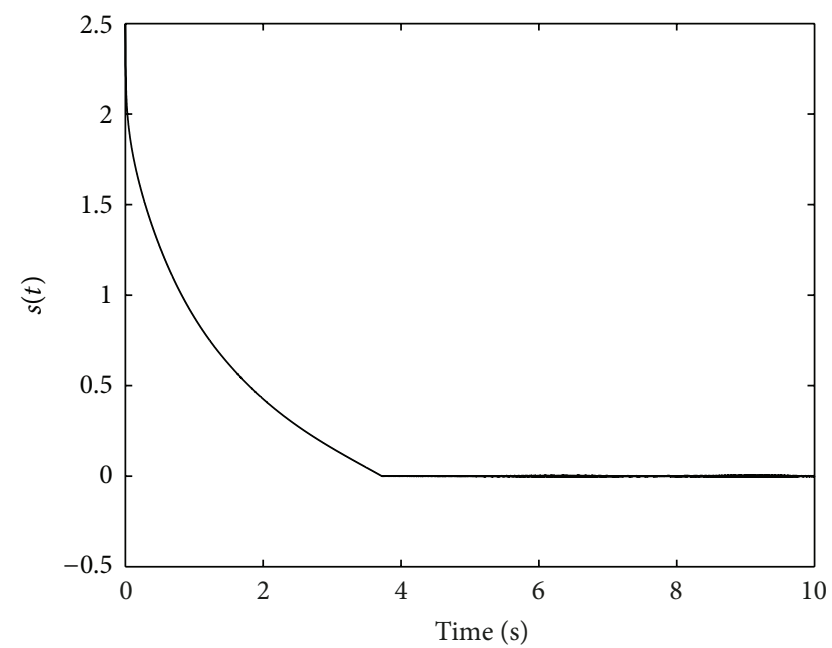

FIgURE 3: Time history of fractional sliding mode with the control input (27).

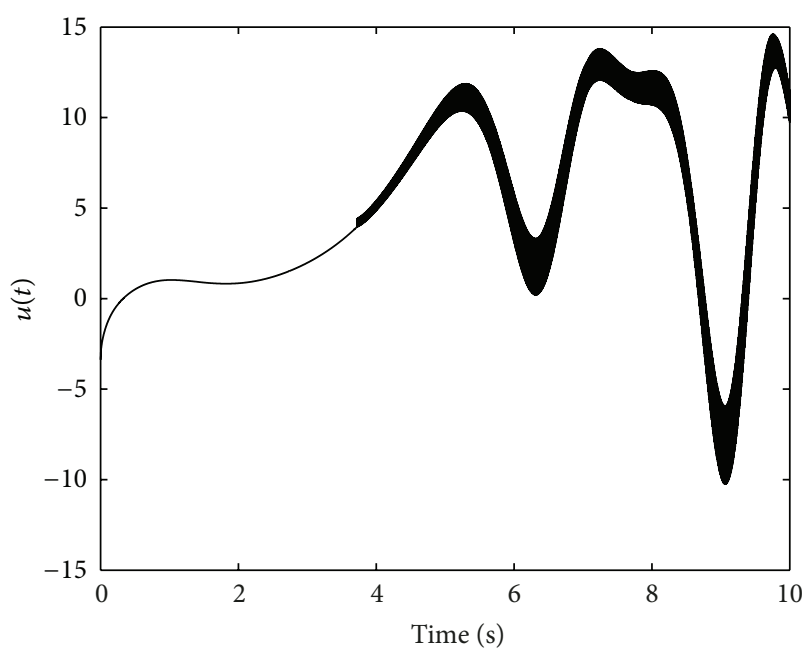

Figure 4: Time history of the control input (27).

synchronization between the fractional Arneodo system and the fractional Genesio-Tesi system. To carry out numerical simulations, we utilize the algorithm for numerical calculation of fractional derivatives introduced in [1]. This method is derived from the Grnwald-Letnikov Definition 1 based on the fact that the three Definitions 1, 2, and 3 are equivalent for a wide class of functions.

The fractional Arneodo system is represented as

$$
\begin{gathered}
D^{\alpha} y_{1}=y_{2}, \\
D^{\alpha} y_{2}=y_{3}, \\
D^{\alpha} y_{3}=5.5 y_{1}-3.5 y_{2}-y_{3}-y_{1}^{3}
\end{gathered}
$$

with the desired tracking signal $y_{d}=y_{1}$.

The fractional Genesio-Tesi system is described as

$$
\begin{gathered}
D^{\alpha} x_{1}=x_{2}, \quad D^{\alpha} x_{2}=x_{3}, \\
D^{\alpha} x_{3}=a(t) x_{1}+b(t) x_{2}+c(t) x_{3}+d(t) x_{1}^{2}+u(t), \\
y=x_{1},
\end{gathered}
$$




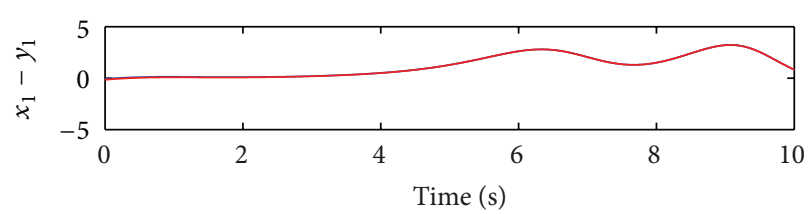

(a)

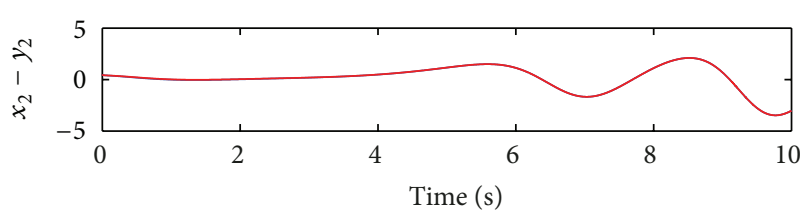

(b)

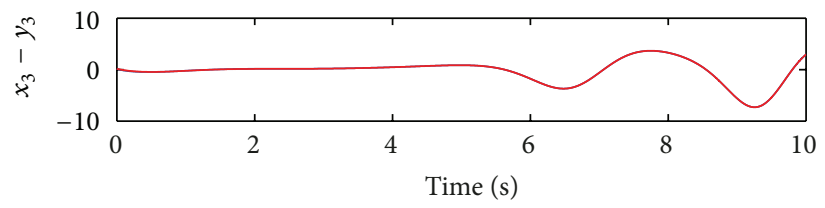

(c)

Figure 5: Synchronization of the fractional Arneodo system and the fractional Genesio-Tesi system with the control input (28) (red line represents the trajectories of the drive system, while blue line represents the trajectories of the response system).

where $a(t), b(t), c(t)$, and $d(t)$ are assumed to be unknown parameters and satisfy $|a(t)+6| \leq 0.1,|b(t)+2.92| \leq 0.1$, $|c(t)+1.2| \leq 0.1$, and $|d(t)-1| \leq 0.1$, from which one has

$$
\begin{gathered}
f(\mathbf{x})=a(t) x_{1}+b(t) x_{2}+c(t) x_{3}+d(t) x_{1}^{2}, \\
\widehat{f}(\mathbf{x})=-6 x_{1}-2.92 x_{2}-1.2 x_{3}+x_{1}^{2}, \\
F(\mathbf{x})=0.1\left(\left|x_{1}\right|+\left|x_{2}\right|+\left|x_{3}\right|+x_{1}^{2}\right) .
\end{gathered}
$$

Both of the fractional Arneodo system (23) and the fractional Genesio-Tesi system (24) exhibit chaos with certain values of fractional order $\alpha$. In the sequel we investigate synchronization between the two fractional chaotic systems using the technique proposed previously. The former system is taken as drive system, while the latter one is considered as response system.

In terms of (11), the sliding surface is constructed as

$$
s=c_{1}\left(x_{1}-y_{1}\right)+c_{2}\left(x_{2}-y_{2}\right)+\left(x_{3}-y_{3}\right),
$$

where $c_{1}=25$ and $c_{2}=10$ satisfy the Routh-Hurwitz conditions [31].

The control law is designed as

$$
\begin{aligned}
u= & 6 x_{1}+2.92 x_{2}+1.2 x_{3}-x_{1}^{2} \\
& +5.5 y_{1}-3.5 y_{2}-y_{3}-y_{1}^{3} \\
& -25\left(x_{2}-y_{2}\right)-10\left(x_{3}-y_{3}\right)-\sigma s \\
& -\sigma s-k \cdot \operatorname{sign}(s) .
\end{aligned}
$$

Initial conditions for the above two systems are, respectively, chosen as $x_{0}=(-0.1,0.5,0.2)$ and $y_{0}=(-0.2,0.5,0.2)$, while parameters in the control law (27) are selected as $\eta=$ 0.1 and $\sigma=0.5$. Numerical simulations of synchronization between the fractional chaotic systems (23) and (24) are presented in Figures 1, 2, 3, and 4, with the simulation time $T_{\text {sim }}=10$ and time step $h=0.0005$. For interpretations of the references to color in these figure legends, the reader is referred to the online version of this paper.

From the simulation results, we see that synchronization performance is excellent but is obtained at the price of high

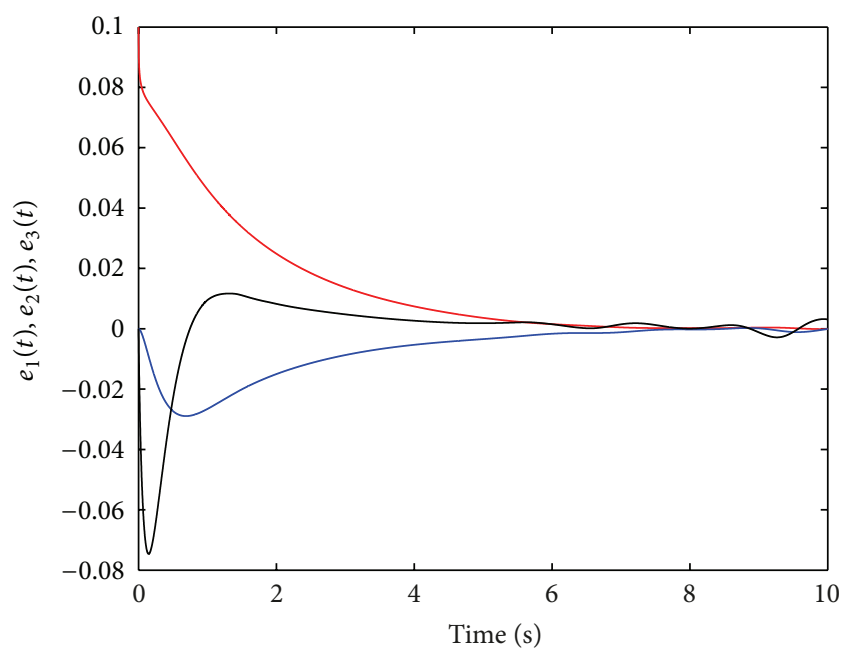

FIgURE 6: Synchronization errors with the control input (28) (red line represents the first error state $e_{1}$, blue line represents the second error state $e_{2}$, and black line represents the third error state $e_{3}$ ).

control chattering. It can be eliminated by replacing the discontinuous switching control law sign $(\cdot)$ by the smooth function $\tanh (\cdot)$ in $(27)$; that is,

$$
\begin{aligned}
u= & 6 x_{1}+2.92 x_{2}+1.2 x_{3}-x_{1}^{2} \\
& +5.5 y_{1}-3.5 y_{2}-y_{3}-y_{1}^{3} \\
& -25\left(x_{2}-y_{2}\right)-10\left(x_{3}-y_{3}\right) \\
& -\sigma s-k \cdot \tanh (s) .
\end{aligned}
$$

Numerical simulations with the modified control law (28) are presented in Figures 5, 6, 7, and 8. For interpretations of the references to color in these figure legends, the reader is referred to the online version of this paper.

From the above simulation results, one can easily see that the fractional Arneodo system and the fractional GenesioTesi system can be effectively synchronized via the proposed sliding mode control technique. Furthermore, the control 


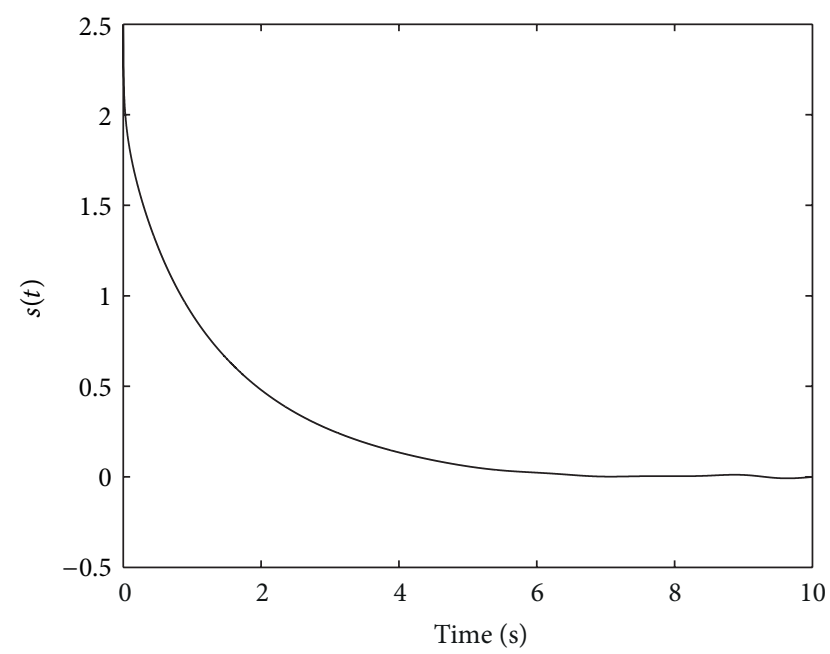

FIGURE 7: Time history of fractional sliding mode with the control input (28).

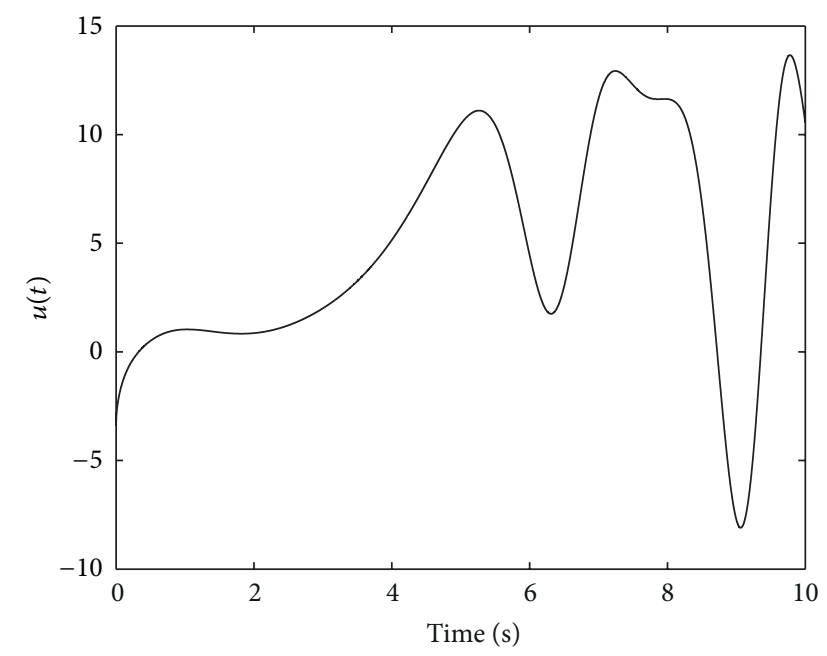

FIGURE 8: Time history of the control input (28).

chattering caused by the discontinuous control law (17) is successfully eliminated by the modification of (28).

\section{Concluding Remarks}

In this paper, we have investigated the pseudo-state sliding control design for fractional SISO nonlinear systems with model inaccuracies. A stable fractional sliding mode surface has been constructed based on the Routh-Hurwitz conditions for fractional differential equations. Then, a sliding mode control law is designed using the Mittag-Leffler stability theorem. Finally, numerical simulations of synchronization of the fractional Arneodo system and the fractional Genesio-Tesi system have been performed to demonstrate the effectiveness of the proposed control technique. be on,

As for the future perspectives, our research activities will

(i) designing adaptive sliding control to deal with parametric uncertainties in $f(\cdot)$, (ii) generalizing the method to fractional MIMO nonlinear systems,

(iii) generalizing the method to incommensurate nonlinear systems.

\section{Conflict of Interests}

The authors do not have any possible conflict of interests.

\section{References}

[1] I. Petráš, Fractional-Order Nonlinear Systems: Modeling, Analysis and Simulation, Springer, Berlin, Germany, 2011.

[2] R. Caponetto, Fractional Order Systems: Modeling and Control Applications, vol. 72, World Scientific Publishing Company, Singapore, 2010.

[3] I. Podlubny, Fractional Differential Equations: An Introduction to Fractional Derivatives, Fractional Differential Equations, to Methods of Their Solution and Some of Their Applications, vol. 198 of Mathematics in Science and Engineering, Academic Press, San Diego, Calif, USA, 1999.

[4] B. J. Lurie, "Three-parameter tunable tilt-integral-derivative (TID) controller," US patent no. 5371670, 1994.

[5] A. Oustaloup, X. Moreau, and M. Nouillant, "The crone suspension," Control Engineering Practice, vol. 4, no. 8, pp. 1101-1108, 1996.

[6] I. Podlubny, "Fractional-order systems and $P I^{\lambda} D^{\mu}$-controllers," IEEE Transactions on Automatic Control, vol. 44, no. 1, pp. 208214, 1999.

[7] H.-F. Raynaud and A. Zergaïnoh, "State-space representation for fractional order controllers," Automatica, vol. 36, no. 7, pp. 1017-1021, 2000.

[8] D. Xue and Y. Q. Chen, "A comparative introduction of four fractional order controllers," in Proceedings of the 4th World Congress on Intelligent Control and Automation, vol. 4, pp. 32283235, June 2002.

[9] S. Dadras and H. R. Momeni, "Control of a fractional-order economical system via sliding mode," Physica A, vol. 389, no. 12, pp. 2434-2442, 2010.

[10] C. Yin, S. Zhong, and W. Chen, "Design of sliding mode controller for a class of fractional-order chaotic systems," Communications in Nonlinear Science and Numerical Simulation, vol. 17, no. 1, pp. 356-366, 2012.

[11] A. Razminia and D. Baleanu, "Complete synchronization of commensurate fractional order chaotic systems using sliding mode control," Mechatronics, vol. 23, no. 7, pp. 873-879, 2013.

[12] J. Yuan, B. Shi, and W. Ji, "Adaptive sliding mode control of a novel class of fractional chaotic systems," Advances in Mathematical Physics, vol. 2013, Article ID 576709, 13 pages, 2013.

[13] M. P. Aghababa, "Robust stabilization and synchronization of a class of fractional-order chaotic systems via a novel fractional sliding mode controller," Communications in Nonlinear Science and Numerical Simulation, vol. 17, no. 6, pp. 2670-2681, 2012.

[14] D. M. Senejohnny and H. Delavari, "Active sliding observer scheme based fractional chaos synchronization," Communications in Nonlinear Science and Numerical Simulation, vol. 17, no. 11, pp. 4373-4383, 2012.

[15] M. P. Aghababa, "Finite-time chaos control and synchronization of fractional-order nonautonomous chaotic (hyperchaotic) 
systems using fractional nonsingular terminal sliding mode technique," Nonlinear Dynamics, vol. 69, no. 1-2, pp. 247-261, 2012.

[16] S. Dadras and H. R. Momeni, "Fractional terminal sliding mode control design for a class of dynamical systems with uncertainty," Communications in Nonlinear Science and Numerical Simulation, vol.17, no. 1, Article ID ZBL1248.93040, pp. 367-377, 2012.

[17] A. Si-Ammour, S. Djennoune, and M. Bettayeb, "A sliding mode control for linear fractional systems with input and state delays," Communications in Nonlinear Science and Numerical Simulation, vol. 14, no. 5, pp. 2310-2318, 2009.

[18] M. R. Faieghi, H. Delavari, and D. Baleanu, "A note on stability of sliding mode dynamics in suppression of fractional-order chaotic systems," Computers \& Mathematics with Applications, vol. 66, no. 5, pp. 832-837, 2013.

[19] M. Pourmahmood, S. Khanmohammadi, and G. Alizadeh, "Synchronization of two different uncertain chaotic systems with unknown parameters using a robust adaptive sliding mode controller," Communications in Nonlinear Science and Numerical Simulation, vol. 16, no. 7, pp. 2853-2868, 2011.

[20] R. Zhang and S. Yang, "Robust synchronization of two different fractional-order chaotic systems with unknown parameters using adaptive sliding mode approach," Nonlinear Dynamics, vol. 71, no. 1-2, pp. 269-278, 2013.

[21] J. Yuan, B. Shi, W. Ji, and T. Pan, "Sliding mode control of the fractional order unified chaotic system," Abstract and Applied Analysis. In press.

[22] B. M. Vinagre, I. Petráš, I. Podlubny, and Y. Q. Chen, "Using fractional order adjustment rules and fractional order reference models in model-reference adaptive control," Nonlinear Dynamics, vol. 29, no. 1-4, pp. 269-279, 2002.

[23] S. Ladaci and A. Charef, "On fractional adaptive control," Nonlinear Dynamics, vol. 43, no. 4, pp. 365-378, 2006.

[24] Z. M. Odibat, "Adaptive feedback control and synchronization of non-identical chaotic fractional order systems," Nonlinear Dynamics, vol. 60, no. 4, pp. 479-487, 2010.

[25] C. Li and Y. Tong, "Adaptive control and synchronization of a fractional-order chaotic system," Pramana, vol. 80, no. 4, pp. 583-592, 2013.

[26] L. Chen, S. Wei, Y. Chai, and R. Wu, "Adaptive projective synchronization between two different fractional-order chaotic systems with fully unknown parameters," Mathematical Problems in Engineering, vol. 2012, Article ID 916140, 16 pages, 2012.

[27] O. P. Agrawal, "A general formulation and solution scheme for fractional optimal control problems," Nonlinear Dynamics, vol. 38, no. 1-4, pp. 323-337, 2004.

[28] Z. D. Jelicic and N. Petrovacki, "Optimality conditions and a solution scheme for fractional optimal control problems," Structural and Multidisciplinary Optimization, vol. 38, no. 6, pp. 571581, 2009.

[29] S. Djennoune and M. Bettayeb, "Optimal synergetic control for fractional-order systems," Automatica, vol. 49, no. 7, pp. 22432249, 2013.

[30] M. Li, D. Li, J. Wang, and C. Zhao, "Active disturbance rejection control for fractional-order system," ISA Transactions, vol. 52, no. 3, pp. 365-374, 2013.

[31] E. Ahmed, A. M. A. El-Sayed, and H. A. A. El-Saka, "On some Routh-Hurwitz conditions for fractional order differential equations and their applications in Lorenz, Rössler, Chua and Chen systems," Physics Letters A, vol. 358, no. 1, pp. 1-4, 2006. 


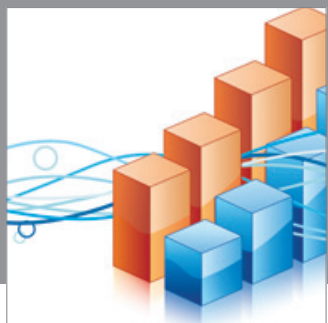

Advances in

Operations Research

mansans

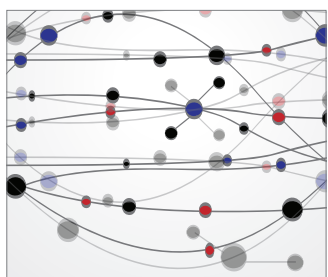

The Scientific World Journal
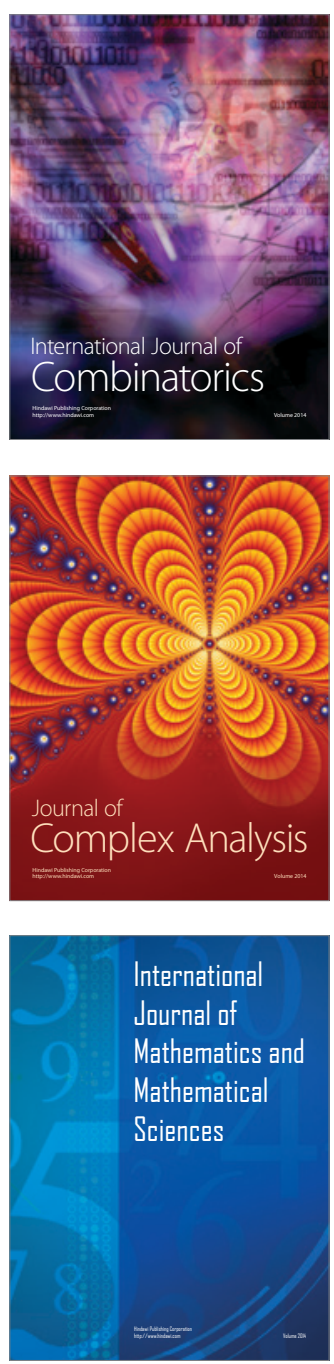
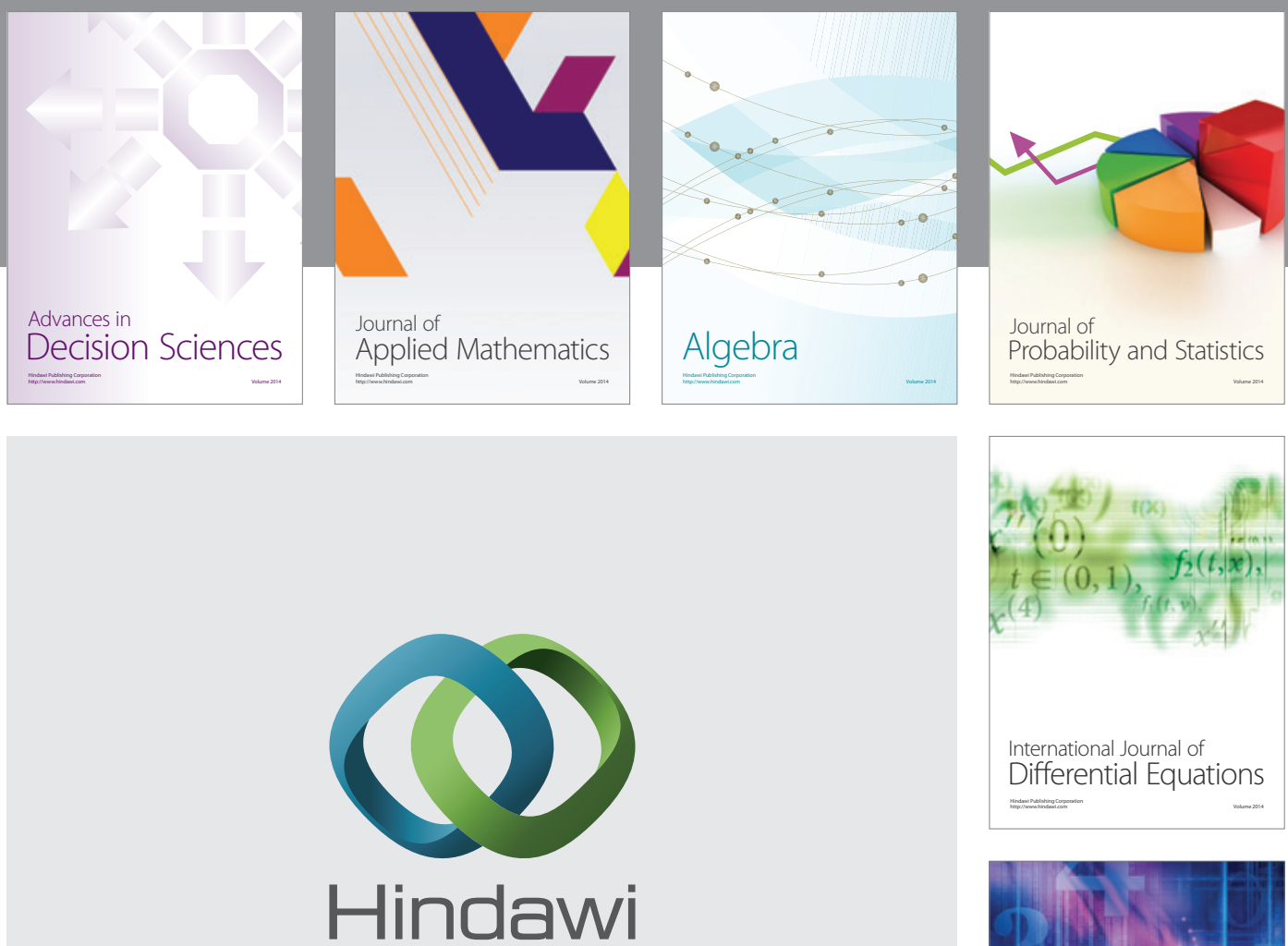

Submit your manuscripts at http://www.hindawi.com
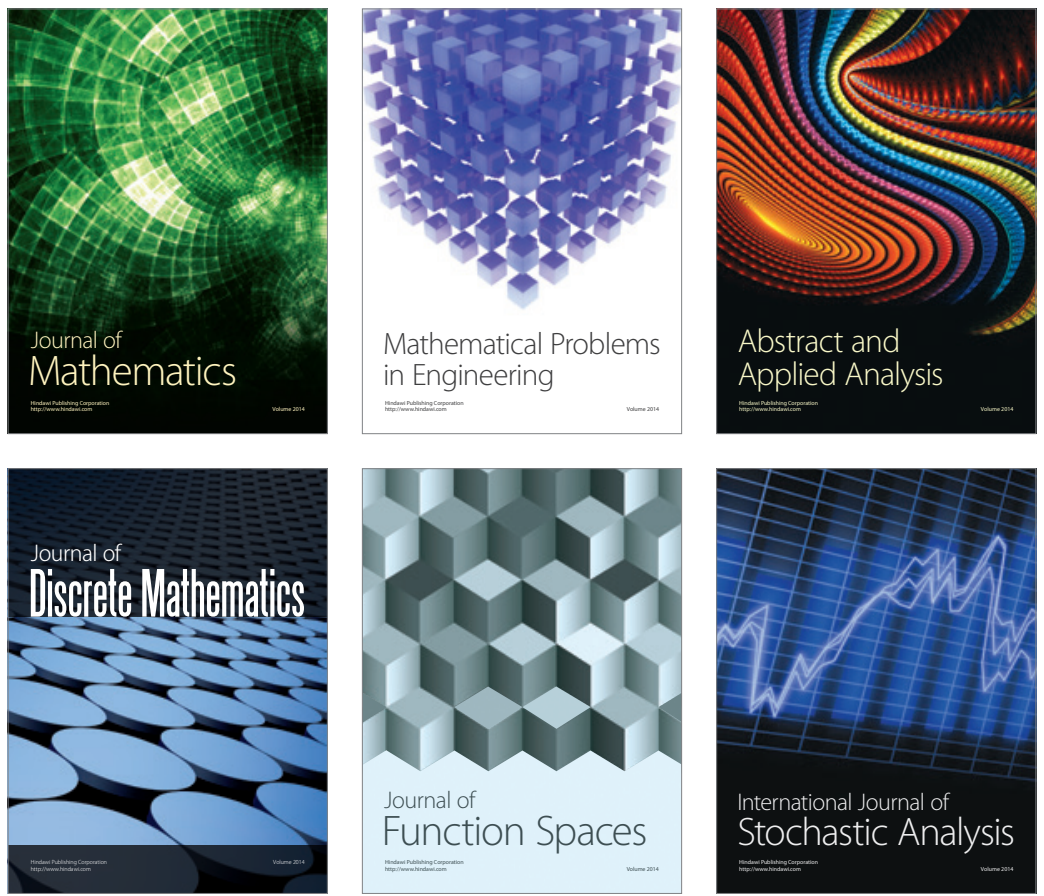

Journal of

Function Spaces

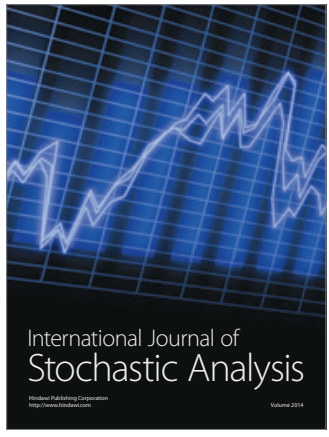

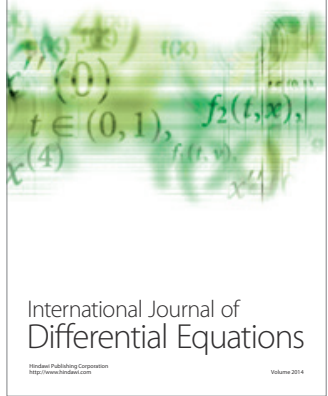
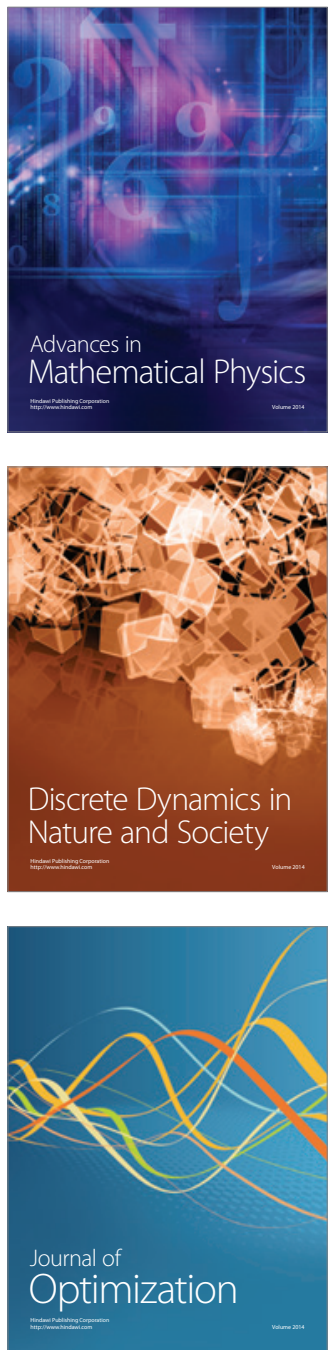